\title{
TALLIP Perspectives: Editorial Commentary
}

The Broadened Focus of the Journal

As I noted in my editorial in the 13(3) issue of the Transactions on Asian Language Information Processing, the Association for Computing Machinery in 2014 approved an extension of the charter of TALIP. In addition to covering speech and language technology applied to Asian languages, we now also cover low-resource languages that are native languages of Africa, Australasia-Oceania, and the Americas. Indeed, nearly all of the native languages of these regions are low-resource, and a large number of them are endangered, many critically so. Concomitant with the expansion of focus is a name change: Transactions on Asian and Low-Resource Language Information Processing (TALLIP).

While this is an expansion of focus, it is nonetheless still within the spirit of the original charter of TALIP. When TALIP was started in 2002, language and speech processing for Asian languages, beyond the big three (Chinese, Japanese, and Korean) was not so common. Now there is quite a bit more work on languages of South and Southeast Asia, as well as substantially more work on West Asian languages such as Arabic. By providing a venue specifically devoted to publishing work on these languages, TALIP has in a small way helped the development of the field.

One of the reasons that work on many Asian languages has been held back is that they, too, are low-resource. Large publicly available corpora of any kind still do not exist for most languages of South Asia and Southeast Asia. Some of these languages, even those spoken by tens of millions of people, do not have a substantial Web presence. Yet we are starting to see more and more interest in these low-resource languages that were already within the purview of TALIP. Extending the focus of the journal to lowresource languages from elsewhere in the world thus comes fairly naturally. And since, as noted, many are not only low in data resources, but also have a dwindling number of speakers, the expanded journal will serve as an outlet for work that aims to preserve as best as possible what lamentably is a dying part of the human experience.

To that end I have invited two new Associate Editors, Steven Bird and Harald Hammarström, both of whom have extensive experience in computational linguistics and in the analysis and documentation of languages that will probably not be with us much longer. TALLIP is now open to submissions that report on any work involving speech or language technology applied to low-resource languages of Africa, Australasia-Oceania, and the Americas.

Richard Sproat

Editor-in-Chief

(C) 2015 ACM 2375-4699/2015/01-ART5 $\$ 15.00$

DOI : http://dx.doi.org/10.1145/2710043 